\title{
Mapping of Regional Failures After Definitive Radiotherapy in Patients with Locally Advanced Cervical Esophageal Carcinoma
}

This article was published in the following Dove Press journal: Cancer Management and Research

\section{Dan Zhao (ID \\ Baomin Zheng \\ Shaowen Xiao \\ Weixin Liu \\ Xiaolong $\mathrm{Xu}$ \\ Huiming Yu \\ Yan Sun \\ Weihu Wang}

Key Laboratory of Carcinogenesis and Translational Research (Ministry of Education/Beijing), Department of Radiation Oncology, Peking University Cancer Hospital Institute, Beijing, People's Republic of China
Correspondence: Weihu Wang; Yan Sun Key Laboratory of Carcinogenesis and Translational Research (Ministry of Education/Beijing), Department of Radiation Oncology, Beijing Cancer Hospital \& Institute, 52\# Fucheng Road, Haidian District, Beijing 100142, People's Republic of China

$\mathrm{Tel} / \mathrm{Fax}+86-10-88196120$;

$+86-10-88|962| 7$

Email wangweihu88@163.com;

lisaysun@139.com
Purpose: This study aimed to retrospectively analyze the failure patterns and clinical outcomes in patients with locally advanced cervical esophageal carcinoma (CEC) after definitive radiotherapy (RT), and illustrate the mapping of regional failures.

Patients and Methods: We reviewed 82 patients with CEC confirmed as squamous cell carcinoma who had completed definitive RT from August 2008 to December 2017. Data on clinical characteristics were collected from the medical records system. Patterns of treatment failures and the survival follow-up were analyzed.

Results: The median age was 58 (38-78) years. In 37 patients, the lesions were limited to the cervical esophagus, while in the remaining 45 patients, the disease got beyond the cervical esophagus (pharynx or thoracic esophagus involved). While 10 patients had stage II disease, 72 had stage III disease. The completed median dose for 95\% PGTV and 95\% PTV was 66 Gy and 58 Gy. While the median follow-up was 27.6 months, the median progression-free survival (PFS) and overall survival (OS) was 16.1 and 28.3 months, respectively. The 3 -year PFS and OS was $30.3 \%$ and $45.3 \%$, respectively. Treatment failures were reported in 55 patients, of which $22,8,7,9,2,3$, and 4 patients had developed local, regional, distant, local-regional, regional-distant, local-distant and local-regional-distant failure, respectively. Of the 41 relapsed nodal sites, 28 were located "in-field" whereas 1 was "marginal" and 12 were "out-field". The most frequent regional relapses were at level VIb, IV and the upper-middle mediastinum.

Conclusion: Regional recurrences focused on lower neck and upper-middle mediastinum, and mainly "in-field", after definitive RT in patients with CEC.

Keywords: esophageal neoplasms, prognosis, radiotherapy, recurrence, squamous cell carcinoma

\section{Introduction}

The cervical esophagus is the short part between the lower border of the cricoid cartilage and the suprasternal notch. ${ }^{1}$ Cervical esophageal carcinoma (CEC) is primarily associated with risk factors such as the use of tobacco and alcohol, ${ }^{2}$ and squamous cell carcinoma (SCC) is the predominant histology. CEC accounts for $2-10 \%$ of all esophageal cancers. ${ }^{3}$ Since CEC is often locally advanced at the time of diagnosis, infiltrating nearby anatomical structures such as the trachea, prevertebral fascia, cricoid, thyroid cartilage, or thyroid gland, its treatment differs from that for thoracic esophageal carcinoma. Moreover, patients with CEC often present with lymph node metastases. Radical resection of $\mathrm{CEC}$ often needs 
pharyngo-laryngo-esophagectomy (PLE), ${ }^{4}$ which leads to high morbidity, mortality, and compromised quality of life. ${ }^{5,6}$ Recent developments in chemoradiotherapy (CRT) have challenged the need for such extensive resections, and the concept of function-preservation has been emphasized to improve the quality of life. Therefore, definitive CRT is the standard treatment modality recommended by the National Comprehensive Cancer Network (NCCN) and the European Society for Medical Oncology (ESMO) guidelines. ${ }^{7,8}$ However, there is still no consensus on the doses and target delineations for CEC. Published studies on CEC are mainly retrospective and have not enrolled large populations. In this study, we will analyze the outcomes of patients with CEC treated with definitive radiotherapy (RT).

\section{Patients and Methods}

\section{Patients and Patient Workup}

The Case Recording System was approved to identify the patients diagnosed with CEC in our center from August 2008 to December 2017, retrospectively. During this period, 126 patients with CEC received RT. Of these 126 patients, 44 were excluded from this study for the following reasons: distant metastases $(\mathrm{n}=10)$, received pre-operative or postoperative RT $(\mathrm{n}=19)$, or did not complete the planning prescribed dose $(n=15)$. The remaining 82 patients were included in this study, and all received definitive RT. All the patients had a confirmed pathological diagnosis of SCC. The pretreatment workup included a complete history and physical examination, full biochemical test, complete blood count, upper digestive tract radiography, endoscopy, computed tomography (CT) scans of the neck and thorax, and ultrasonography of the abdominal region and the cervical region with or without fine-needle aspiration cytology when cervical nodal metastasis was detected. In addition, bronchoscopy was performed for patients with locally advanced diseases unless when the patient's condition did not permit or if the patient did not consent. All patients underwent disease staging using the AJCC 6th version staging system because the new staging systems (AJCC 7th and 8th versions) requiring the exact numbers of metastatic nodes are more suitable for postoperative patients. The study protocol was in accordance with the guidelines outlined in the Declaration of Helsinki and was approved by the Ethics Committee of the Beijing Cancer Hospital $\square$ Institute, Peking University
School of Oncology (Document Number: 2019YJZ75). All patients provided written, informed consent.

\section{Treatments}

There is still no consensus on the radiation doses and chemotherapy regimens for CEC by far. Different treatment modalities and chemotherapy regimens were administered to these patients according to age, general status, disease extension, stages and cardiopulmonary diseases, etc. The details are shown in Figure 1.

While 42 patients received induction chemotherapy (IC, taxanes + platinum) before RT, 44 patients received concurrent CRT (CCRT, taxanes \pm platinum, or platinum \pm fluorouracil, or fluorouracil), and 7 patients received concurrent targeted therapy (CTT) with cetuximab or nimotuzumab. Platinum-based adjuvant chemotherapy was given to 12 patients.

Patients were immobilized in a supine position using a thermoplastic head-neck-shoulder mask. They underwent simulation on a dedicated CT scanner, using $3 \mathrm{~mm}$ slices. Intravenous contrast was used unless when the patient's condition did not permit or when the patient did not consent.

All target volumes were outlined on the treatment planning CT images. The gross tumor volume (GTV), divided as primary gross tumor volume (GTVp) and nodal gross tumor volume (GTVnd), included all gross diseases as determined by imaging, clinical, and endoscopic findings. Grossly positive lymph nodes were defined as any para-esophageal lymph node with a short axis diameter $\geq 5 \mathrm{~mm}$, other thoracic lymph nodes with a short axis diameter $\geq 10 \mathrm{~mm}$, extracapsular spread, lymph nodes with obvious necrosis or enhancement, $\geq 3$ lymph nodes existing in a cluster, maximal standard uptake value (SUVmax) $\geq 2.5$ in PET-CT images, or fine-needle aspiration cytology confirmed. The position of each node was assessed based on the Radiation Therapy Oncology Group (RTOG) lymph node definitions. ${ }^{9}$ The clinical target volume (CTV) included the GTVp with an additional radial margin of 5-10 $\mathrm{mm}$ and longitudinal margins of 30-50 mm (distal) and 10-50 mm (proximal) based on the lesion location and hypopharyngeal invasion. The CTV was modified based on anatomical barriers. Nodal regions of GTVnd, if any, were included in the CTV. Elective nodal irradiation (ENI) of the nodal regions was delivered according to the levels of the metastatic lymph nodes. If there was no metastatic lymph node, ENI of the nodal regions was delivered to level IV, V, superior paraesophageal region (VIb) and upper-middle mediastinal region (varied according to extension of thoracic esophagus 


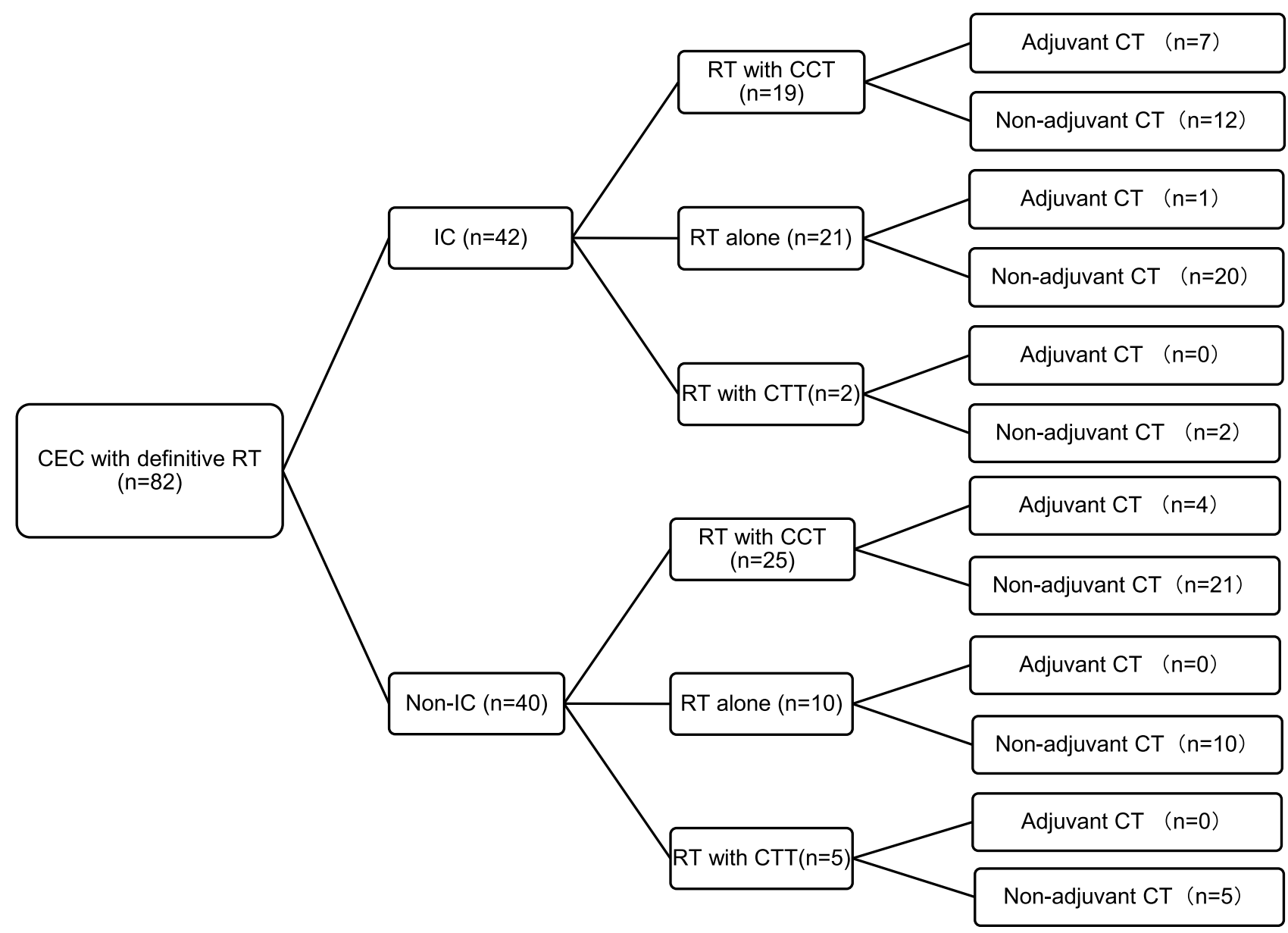

Figure I Treatment modalities.

Abbreviations: CEC, cervical esophageal carcinoma; IC, inductive chemotherapy; RT, radiotherapy; CCT, concurrent chemotherapy; CTT, concurrent target therapy; CT, chemotherapy.

invasion and mediastinal lymph node metastasis). If GTVnd was under the level of cricoid cartilage, nodal regions of ENI should include level III, IV, V, VIb and upper-middle mediastinal region. If any GTVnd was located between the level of hyoid bone and cricoid cartilage, nodal regions of ENI should also include level II. If any GTVnd was located above hyoid bone, upper border of ENI should be elevated to the skull base (VIIb). Retropharyngeal nodal region (VIIa) was included if hypopharynx was involved. The planning GTV (PGTV) and planning target volume (PTV) were generated with a $5 \mathrm{~mm}$ radical margin, and a $5-10 \mathrm{~mm}$ longitudinal margin from GTV and CTV. Organs at risk, including the spinal cord, parotid glands, thyroid gland, lung, heart, larynx, trachea, mandible, and temporomandibular joints were contoured. A $5 \mathrm{~mm}$ margin was added to the spinal cord to form the planning risk volume.

Intensity-modulated radiation therapy (IMRT) with 6-8 MV photon beams was administered to all the patients. The completed prescription dose was 59.4-70 Gy at the periphery of the PGTV at 1.8-2.12 Gy per daily fraction. The median completed dose for the PGTV was 66 Gy. The completed prescription dose was 50-60 Gy at the periphery of the PTV at 1.8-2.0 Gy per daily fraction. The median completed dose of PTV was $58 \mathrm{~Gy}$. At the planning stage, the goal was to cover $\geq 95 \%$ of each target volume with the prescribed dose. All patients were treated with one fraction daily, 5 days per week.

\section{Treatment Monitoring}

All patients were evaluated weekly during the RT and were required to be followed up after the completion of treatment as follows: 1 month after the completion of treatment, every 3 months in the first 2 years, every 6 months from Year 3 to Year 5, and annually thereafter. Each follow-up included a complete examination with a basic blood routine test, serum chemistry, cancer biomarkers, upper digestive tract radiography, CT scans of the neck and thorax, and ultrasonography of the cervical region and the abdominal region. 
Toxicity was scored according to the National Cancer Institute Common Terminology Criteria for Adverse Events (NCI CTC AE) version 3.0.

\section{Definition of Failure Sites}

The records of all patients were reviewed to assess the sites of treatment failures. The response evaluation criteria in solid tumor (RECIST) were used to define all relapses. Failure patterns were classified as local, regional, or distant, and were determined at the time of the last follow-up. Local failure referred to the failure of the primary tumor. Regional failure was defined as recurrence in the regional lymph nodes. Distant failure was defined as the appearance of tumor at a site representing hematogenous dissemination. PET-CT or CT performed at relapses and planning CT performed at baseline were compared and matched, and failure sites were marked on our treatment planning system (TPS, Eclipse, Varian Medical System, Version 15.5). "Out-field" failure was defined if less than $20 \%$ of the volume of failure was inside the $95 \%$ isodose line, "marginal" if $20-95 \%$ of the volume of failure within the $95 \%$ isodose line, or "in-field" if $95 \%$ of the volume of failure was within the $95 \%$ isodose line. ${ }^{10}$

\section{Statistical Analysis}

The Statistical Package for Social Sciences, version 19.0 (SPSS, Chicago, IL), software was used for statistical analysis. The progress-free survival (PFS) and overall survival (OS) were estimated by the Kaplan-Meier method and were measured from the first day of treatment to the date of the event. The Cox proportional hazards model was used to test the independent factors of OS and PFS. All statistical tests were based on a two-sided significance level, and $\mathrm{p}<0.05$ was considered to indicate statistical significance.

\section{Results}

\section{Patient Characteristics}

The clinical characteristics of the patients have been listed in Table 1. The median age of the 82 patients was 58 (38-78) years. In 37 patients, the lesions were limited to the cervical esophagus, while in the remaining 45 patients got the disease beyond the cervical esophagus (pharynx or thoracic esophagus involved). While 10 patients had stage II disease, 72 of them had stage III disease. The completed median dose for 95\% PGTV and 95\% PTV was 66 Gy and 58 Gy, respectively. Six patients were diagnosed with synchronous multiple primary esophageal carcinomas.

\section{Treatment Outcomes}

The median follow-up time was 27.6 months. 55 patients had disease progression, and 51 died. For all patients, the median PFS and OS was 16.1 months and 28.3 months, respectively. The 1, 2, 3, and 5-year PFS was $60.1 \%, 34.9 \%, 30.3 \%$ and $26.0 \%$, respectively. The $1,2,3$, and 5 -year OS was $78.1 \%$, $51.1 \%, 45.3 \%$ and $31.5 \%$, respectively (Figure 2 ).

\section{Failure Patterns}

55 patients had developed treatment failure at their last follow-up visit, of which $22,8,7,9,2,3$, and 4 patients

Table I Clinical Characteristics of Patients with Cervical Esophageal Carcinoma

\begin{tabular}{|c|c|}
\hline Characteristics & n (\%) \\
\hline Median age (year) & 58 \\
\hline$\geq 58$ & $42(51.2)$ \\
\hline$<58$ & $40(48.8)$ \\
\hline \multicolumn{2}{|l|}{ Gender } \\
\hline Male & $63(76.8)$ \\
\hline Female & 19(23.2) \\
\hline \multicolumn{2}{|l|}{ Tumor extension } \\
\hline CE alone & $37(45.1)$ \\
\hline Beyond CE (pharynx or thoracic esophagus invasion) & $45(54.9)$ \\
\hline \multicolumn{2}{|l|}{ Histologic grade } \\
\hline 1 & $6(7.3)$ \\
\hline 2 & $39(47.6)$ \\
\hline 3 & $26(31.7)$ \\
\hline $\mathrm{x}$ & $\mathrm{II}(13.4)$ \\
\hline \multicolumn{2}{|l|}{ T stage } \\
\hline TI & $0(0)$ \\
\hline $\mathrm{T} 2$ & $7(8.5)$ \\
\hline T3 & $14(17.1)$ \\
\hline $\mathrm{T} 4$ & $61(74.4)$ \\
\hline \multicolumn{2}{|l|}{$\mathrm{N}$ stage } \\
\hline No & $10(12.2)$ \\
\hline $\mathrm{NI}$ & $72(87.8)$ \\
\hline \multicolumn{2}{|l|}{ Overall stage (2002 AJCC) } \\
\hline Stage II & $10(12.2)$ \\
\hline Stage III & $72(87.8)$ \\
\hline Median complete 95\%PGTV dose & $66 \mathrm{~Gy}$ \\
\hline Complete 95\%PGTV dose $\geq 66 \mathrm{~Gy}$ & $46(56.1)$ \\
\hline Complete 95\%PGTV dose<66Gy & $36(43.9)$ \\
\hline Median complete 95\%PTV dose & $58 \mathrm{~Gy}$ \\
\hline Complete 95\%PTV dose $\geq 58 \mathrm{~Gy}$ & $43(52.4)$ \\
\hline Complete $95 \% \mathrm{PTV}$ dose $<58 \mathrm{~Gy}$ & $39(47.6)$ \\
\hline
\end{tabular}

Abbreviations: CE, cervical esophagus; AJCC, American Joint Committee on Cancer; PGTV, planning gross tumor volume; PTV, planning target volume. 


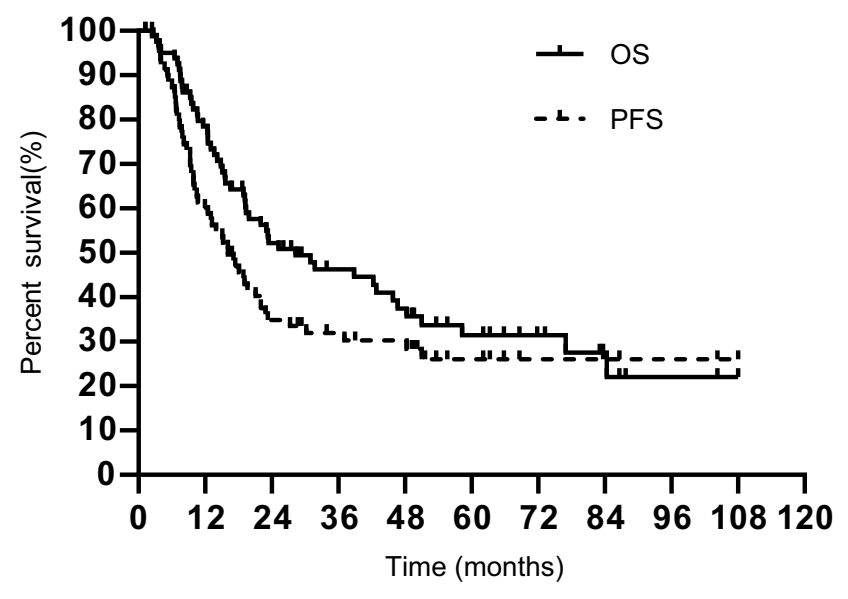

Figure 2 Kaplan-Meier curve of progress-free survival (PFS) and overall survival (OS).

got local only, regional only, distant only, local-regional, regional-distant, local-distant and local-regional-distant failure, respectively. Figure 3 shows the distribution of the sites of failures. Forty-one relapsed nodal sites of 23 patients with regional failures were mapped in detail by the RTOG nodal stations (Figure 4). The most frequent recurrence nodal sites were level VIb, upper-middle mediastinum, IV, III, II, V, retropharyngeal lymph nodes (VIIa) and Ib. Of the 41 nodal sites, 28 were located "in-field" whereas 1 was "marginal" and 12 were "out-field". The distant metastatic sites of 16 patients with distant failure included the lungs, non-regional lymph nodes, bone, liver, and pleural effusion in 10, 6, 3, 3, and 2 patients, respectively.

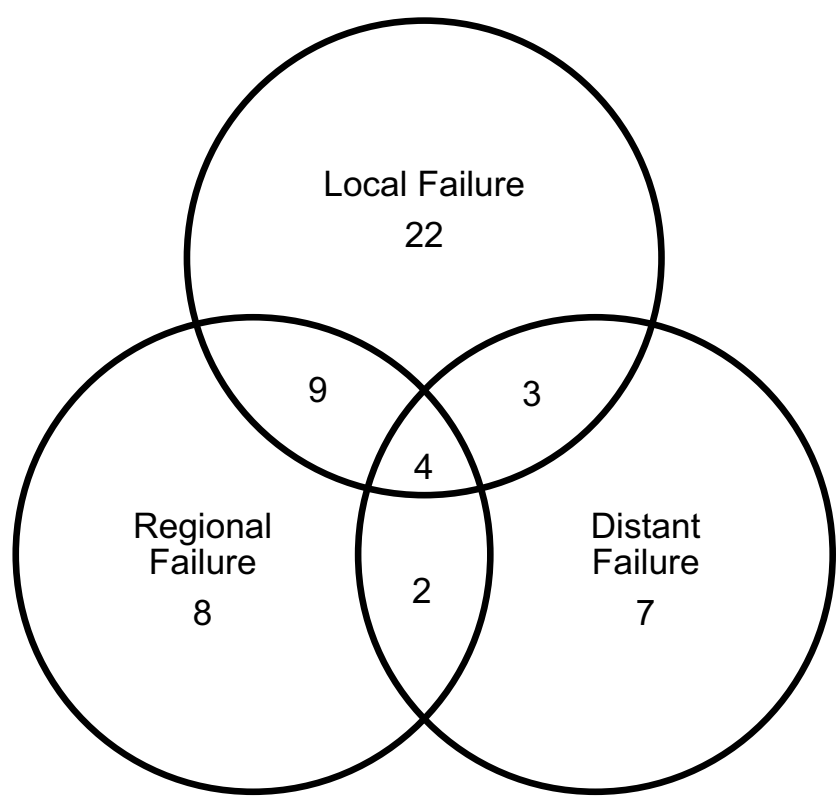

Figure 3 Patterns of failure.

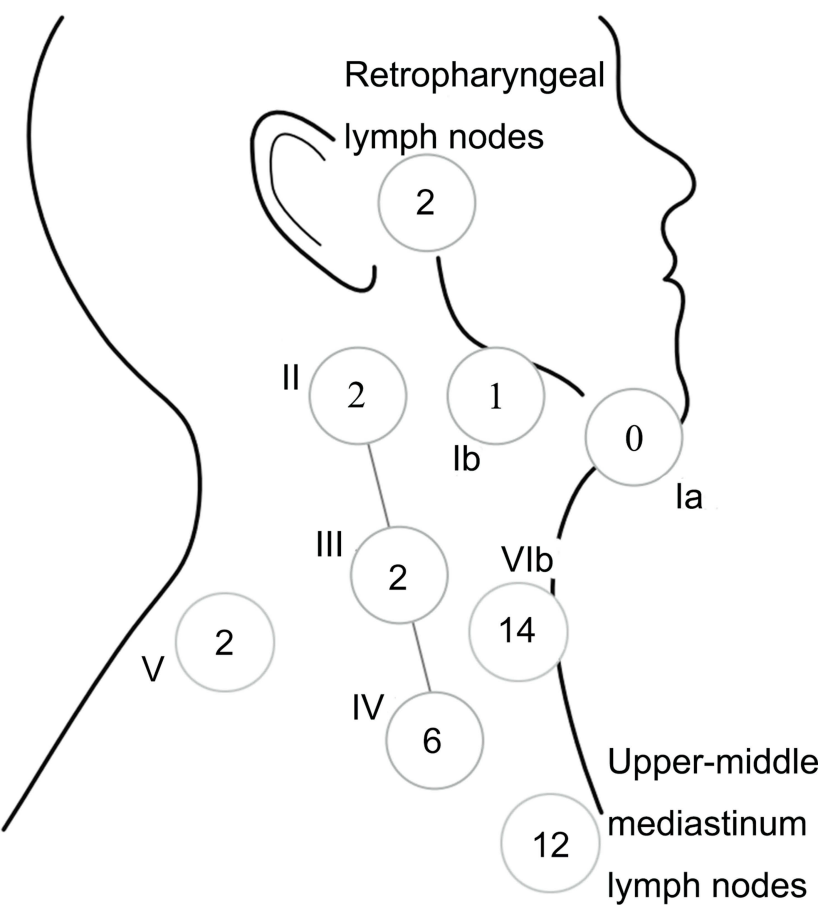

Figure 4 Nodal sites distribution of regional failure.

\section{Toxicities}

The most frequently observed acute toxicity was of grade 1 or 2 . The incidence of acute grade 3 mucositis (including pharyngitis), skin reaction, radiation pneumonia, and leukopenia was $1.2 \%, 1.2 \%, 1.2 \%$, and $19.5 \%$, respectively. Twentyseven patients $(32.9 \%)$ received a nutritional intervention, including parenteral nutrition, naso-gastric/naso-intestinal feeding tube, and percutaneous endoscopy gastrostomy or jejunostomy. Five patients developed fistula after completion of RT, because of tumor penetration of esophageal wall and quick tumor regression after RT.

\section{Discussion}

In the present study, analysis of 82 patients with $\mathrm{CEC}$ treated with definitive RT showed that the 1, 2, 3, and 5-year PFS was $60.1 \%, 34.9 \%, 30.3 \%$, and $26.0 \%$, respectively and the $1,2,3$, and 5-year OS was $78.1 \%, 51.1 \%$, $45.3 \%$, and $31.5 \%$, respectively. In previous studies, ${ }^{11,19}$ the 2, 3, 5-year OS rates in patients who were treated with RT were $35.0-52.0 \%, 29.0-39.3 \%$, and $18.6-55.0 \%$, respectively, which are in line with our findings.

Tong et al have reported the outcomes in 62 patients who underwent PLE as the primary treatment and 21 patients who received up-front CRT. ${ }^{5}$ The 2-year OS in the PLE and CRT groups was $37.6 \%$ and $46.9 \%$, respectively $(p=0.390) .{ }^{5}$ Other studies have reported that the 
5-year OS following surgery only for CEC is $24.0-47.0 \%,{ }^{20,26}$ which is comparable to the OS following definitive RT (18.6-55.0\%). ${ }^{11,19}$

A study by Cao et al including 115 patients with CEC treated with definitive RT (80 with RT alone and 35 with CCRT) has reported that the 2-year OS was significantly better in the patients receiving a radiation dose $\geq 66$ Gy than in the patients receiving a dose $<66$ Gy $(55.6 \%$ vs $37.5 \%$, p $=$ $0.018) .{ }^{19}$ However, a study by Gkika et al $(\mathrm{n}=55$, CEC treated with CCRT) also suggested that a higher radiation dose $(>60$ Gy vs $\leq 60 \mathrm{~Gy}$ ) did not lead to better 5-year OS (24\% vs $26 \%$, $\mathrm{p}=0.78) .{ }^{16}$ In our previous study ${ }^{27}$ including 97 patients with CEC who received doses of 95\% PGTV 50-70 Gy (median 66 Gy) and 95\% PTV 40-60 Gy (median 55 Gy) because of adverse reactions, we also arrived at the same conclusion as Cao et $\mathrm{al}^{19}$, that a higher $95 \%$ PGTV dose ( $\geq 66 \mathrm{~Gy}$ ) improved the OS. While, in our current study, when the completed doses for 95\% PGTV and 95\% PTV were raised to 59.4-70 Gy (median 66 Gy) and 50-60 Gy (median 58 Gy) respectively, we could not confirm the beneficial effects of higher dose radiation (shown in Appendix Figure 1). This discordance indicated that the minimum dose of definitive RT for CEC might be 60 Gy for 95\% PGTV and 50 Gy for 95\% PTV. This speculation needs to be further verified.

The incidence of lymph node metastasis in our study was $87.8 \%$, which was higher than that reported in other published articles (48.0-68.8\%). ${ }^{13,15,16,19}$ The metastatic lymph nodes in our study were mostly at levels VIb, the upper mediastinum, II, III and IV, which was in accordance with published data. ${ }^{28,31}$ Additionally, the most frequent recurrences of regional lymph nodes were at levels VIb, the upper-middle mediastinum and IV. The "outfield" failure accounted for $29.27 \%$ (12/41) of the regional recurrences in our study. Given the high rates and extensive levels of lymph node metastasis, ENI has been implemented by majority of the investigation centers. ${ }^{11,18}$

The potential limitations of our study include the retrospective nature of the analysis, the involvement of a single-institution, and the relatively small sample size. Our results should ideally be tested in a larger database or a prospective clinical trial.

\section{Conclusion}

The results of this study suggested that the dose escalation and ENI for CEC may still be beneficial. The failure patterns in CEC patients after definitive RT mainly indicated locoregional recurrence. The most frequent regional recurrences in our study were at levels VIb, IV and upper-middle mediastinum. Target volumes for ENI and the optimal dose of RT need to be further clarified in prospective trials.

\section{Funding}

This work was supported by grants from the Beijing Municipal Science \& Technology Commission No. Z181100001718192, Beijing Natural Science Foundation No. 7182028, and the Science Foundation of Peking University Cancer Hospital No.18-03.

\section{Disclosure}

The authors have declared no conflicts of interest in this work.

\section{References}

1. Rice TW, Ishwaran H, Ferguson MK, Blackstone EH, Goldstraw P. Cancer of the esophagus and esophagogastric junction: an eighth edition staging primer. $J$ Thorac Oncol. 2017;12:36-42. doi:10.1016/j.jtho.2016.10.016

2. Popescu CR, Bertesteanu SV, Mirea D, Grigore R, Ionescu D, Popescu B. The epidemiology of hypopharynx and cervical esophagus cancer. J Med Life. 2010;3:396-401.

3. Lee DJ, Harris A, Gillette A, et al. Carcinoma of the cervical esophagus: diagnosis, management, and results. South Med J. 1984;77:1365-1367. doi:10.1097/00007611-198411000-00004

4. Wei WI, Lam LK, Yuen PW, et al. Current status of pharyngolaryngoesophagectomy and pharyngogastric anastomosis. Head Neck. 2015;20:240-244. doi:10.1002/(SICI)1097-0347(199805)20:3<240:: AID-HED9 $>3.0 . \mathrm{CO} ; 2-0$

5. Tong DK, Law S, Kwong DL, et al. Current management of cervical esophageal cancer. World J Surg. 2011;35:600-607. doi:10.1007/ s00268-010-0876-7

6. Peracchia A, Bonavina L, Botturi M, et al. Current status of surgery for carcinoma of the hypopharynx and cervical esophagus. Dis Esophagus. 2001;14:95-97. doi:10.1046/j.1442-2050.2001.00163.x

7. National comprehensive cancer network. Clinical practice guidelines in oncology (NCCN guidelines). Esophageal and esophagogastric junction cancers. NCCN.org.

8. Stahl M, Mariette C, Haustermans K, Cervantes A, Arnold D, European Society for Medical Oncology: clinical practice guidelines. Oesophageal cancer: ESMO clinical practice guidelines for diagnosis, treatment and follow-up. Ann Oncol. 2013;24(Supp16):vi51-56. doi:10.1093/annonc/mdt342

9. Grégoire V, Ang K, Budach W, et al. Delineation of the neck node levels for head and neck tumors: a 2013 update. DAHANCA, EORTC, HKNPCSG, NCIC CTG, NCRI, RTOG, TROG consensus guidelines. Radiother Oncol. 2014;110:172-181. doi:10.1016/j.radonc.2013.10.010

10. Dawson LA, Anzai Y, Marsh L, et al. Patterns of local-regional recurrence following parotid-sparing conformal and segmental intensity-modulated radiotherapy for head and neck cancer. Int J Radiat Oncol Biol Phys. 2000;46:1117-1126. doi:10.1016/ S0360-3016(99)00550-7

11. Burmeister BH, Dickie G, Smithers BM, et al. Thirty-four patients with carcinoma of the cervical esophagus treated with chemoradiation therapy. Arch Otolaryngol Head Neck Surg. 2000;126:205-208. doi:10.1001/archotol.126.2.205

12. Wang S, Liao Z, Chen Y, et al. Esophageal cancer located at the neck and upper thorax treated with concurrent chemoradiation: a single-institution experience. $J$ Thorac Oncol. 2006;1:252-259. doi:10.1016/S1556-0864(15)31576-8 
13. Yamada K, Murakami M, Okamoto Y, et al. Treatment results of radiotherapy for carcinoma of the cervical esophagus. Acta Oncol. 2006;45:1120-1125. doi:10.1080/02841860600609768

14. Uno T, Isobe K, Kawakami H, et al. Concurrent chemoradiation for patients with squamous cell carcinoma of the cervical esophagus. Dis Esophagus. 2007;20:12-18. doi:10.1111/j.1442-2050.2007.00632.x

15. Huang SH, Lockwood G, Brierley J, et al. Effect of concurrent high-dose cisplatin chemotherapy and conformal radiotherapy on cervical esophageal cancer survival. Int J Radiat Oncol Biol Phys. 2008;71:735-740. doi:10.1016/j.ijrobp.2007.10.022

16. Gkika E, Gauler T, Eberhardt W, et al. Long-term results of definitive radiochemotherapy in locally advanced cancers of the cervical esophagus. Dis Esophagus. 2014;27:678-684. doi:10.1111/dote.12146

17. Ludmir EB, Palta M, Zhang X, et al. Incidence and prognostic impact of high-risk HPV tumor infection in cervical esophageal carcinoma. $J$ Gastrointest Oncol. 2014;5(6):401-407. doi:10.3978/j.issn.20786891.2014.053

18. Zhang P, Xi M, Zhao L, et al. Clinical efficacy and failure pattern in patients with cervical esophageal cancer treated with definitive chemoradiotherapy. Radiother Oncol. 2015;116:257-261. doi:10.1016/j. radonc.2015.07.011

19. Cao C, Luo J, Gao L, et al. Definitive radiotherapy for cervical esophageal cancer. Head Neck. 2015;37:151-155. doi:10.1002/hed.23572

20. Miyata H, Takahashi T, Kurokawa Y, et al. Larynx-preserving limited resection and free jejunal graft for carcinoma of the cervical esophagus. World J Surg. 2013;37:551-557. doi:10.1007/s00268-012-1875-7

21. Sun F, Li X, Lei D, et al. Surgical management of cervical esophageal carcinoma with larynx preservation and reconstruction. Int J Clin Exp Med. 2014;7:2771-2778.

22. Ott K, Lordick F, Molls M, et al. Limited resection and free jejunal graft interposition for squamous cell carcinoma of the cervical esophagus. Br J Surg. 2009;96:258-266. doi:10.1002/bjs.6437
23. Daiko H, Hayashi R, Sakuraba M, et al. Surgical management of carcinoma of the cervical esophagus. J Surg Oncol. 2007;96:166-172. doi:10.1002/jso.20795

24. Triboulet JP, Mariette C, Chevalier D, Amrouni H. Surgical management of carcinoma of the hypopharynx and cervical esophagus: analysis of 209 cases. Arch Surg. 2001;136:1164-1170. doi:10.1001/ archsurg.136.10.1164

25. Jiang M, He X, Wu D, et al. Reconstruction techniques for hypopharyngeal and cervical esophageal carcinoma. $J$ Thorac Dis. 2015;7:449-454. doi:10.3978/j.issn.2072-1439.2015.02.12

26. Wang HW, Chu PY, Kuo KT, et al. A reappraisal of surgical management for squamous cell carcinoma in the pharyngoesophageal junction. J Surg Oncol. 2006;93:468-476. doi:10.1002/jso.20472

27. Zhao D, Zheng BM, Xiao SW, et al. Patterns of failure and clinical outcomes of radiotherapy for cervical esophageal carcinoma. Chin J Radiol Med Prot. 2019;39:44-50.

28. Hirano S, Nagahara K, Moritani S, et al. Upper mediastinal node dissection for hypopharyngeal and cervical esophageal carcinomas. Ann Otol Rhinol Laryngol. 2007;116:290-296. doi:10.1177/ 000348940711600413

29. Martins AS. Neck and mediastinal node dissection in pharyngolaryngoesophageal tumors. Head Neck. 2001;23:772-779. doi:10.1002/ hed. 1110

30. Timon CV, Toner M, Conlon BJ. Paratracheal lymph node involvement in advanced cancer of the larynx, hypopharynx, and cervical esophagus. Laryngoscope. 2003;113:1595-1599. doi:10.1097/ 00005537-200309000-00035

31. Weber RS, Marvel J, Smith P, et al. Paratracheal lymph node dissection for carcinoma of the larynx, hypopharynx, and cervical esophagus. Otolaryngol Head \& Neck Surg. 1993;108:11-17. doi:10.1177/ 019459989310800102

\section{Publish your work in this journal}

Cancer Management and Research is an international, peer-reviewed open access journal focusing on cancer research and the optimal use of preventative and integrated treatment interventions to achieve improved outcomes, enhanced survival and quality of life for the cancer patient.
The manuscript management system is completely online and includes a very quick and fair peer-review system, which is all easy to use. Visit http://www.dovepress.com/testimonials.php to read real quotes from published authors. 